\title{
Silicon-photonics-based optical frequency synthesizer
}

\author{
Neetesh Singh ${ }^{1 *}$, Ming Xin ${ }^{1}$, Nanxi Li ${ }^{1,2}$, Diedrik Vermeulen${ }^{1}$, Alfonso Ruocco' ${ }^{1}$, Emir Salih Magden ${ }^{1}$, Katia \\ Shtyrkova $^{1}$, Patrick T. Callahan ${ }^{1}$, Christopher Baiocco ${ }^{3}$, Erich Ippen ${ }^{1}$, Franz X. Kärtner ${ }^{1,4}$, and Michael R. \\ Watts $^{1}$ \\ ${ }^{1}$ Research Laboratory of Electronics, Massachusetts Institute of Technology, 77 Massachusetts Avenue, Cambridge, MA 02139, USA \\ ${ }^{2} J o h n$ A. Paulson School of Engineering and Applied Science, 29 Oxford Street, Harvard University, Cambridge, MA 02138, USA \\ ${ }^{3}$ College of Nanoscale Science and Engineering, SUNY Polytechnic Institute, 257 Fuller Road, Albany, NY 12203, USA \\ ${ }^{4}$ Centre for Free Electron Laser Science (CFEL)-DESY and University of Hamburg, Notkestrasse 85, 22607 Hamburg, Germany \\ *Corresponding author: neeteshs@mit.edu
}

\begin{abstract}
We demonstrate silicon-photonics-based octave spanning optical frequency combs phase coherently locked to a microwave oscillator for optical frequency synthesis. This system offers capability for precision optical synthesis of CW laser over the entire C-band.

OCIS codes: (190.7110) Ultrafast nonlinear optics; (130.0130) Integrated optics; (320.6629) Supercontinuum generation.
\end{abstract}

Optical frequency combs that phase coherently build a link from optical frequencies down to electronically measurable radio frequencies have been proven extremely useful for applications including frequency metrology and optical atomic clocks [1]. Such systems enabled precision frequency synthesis, where any CW laser oscillator can be coherently stabilized and tuned over the wide bandwidth of a phase-locked frequency comb, thus synthesizing precise optical frequencies on demand [2]. For systems to be widely available at low cost they should be manufacturable in CMOS foundries. Here we demonstrate our progress towards such systems using a silicon-on-insulator platform. We show octave spanning coherent and phase locked (to a microwave reference) comb generation by detecting and stabilizing the pulse repetition rate $\left(f_{\text {rep }}\right)$ and carrier offset frequency $\left(f_{\text {ceo }}\right)$ of a mode-locked laser (MLL), which is used for supercontinuum (SC) generation and $\mathrm{f}-2 \mathrm{f}$ conversion with the help of second harmonic generation (SHG) in a silicon waveguide. Subsequently, a low noise integrated CW laser is phase locked in the C-band to the stabilized frequency comb for optical frequency synthesis.

A commercial MLL with a repetition rate of $200 \mathrm{MHz}, 18 \mathrm{pJ}$ of coupled energy and pulse width $>50 \mathrm{fs}$ is used to pump the SC waveguide at $1.9 \mu \mathrm{m}$, as shown in Fig.1. The SC is generated by designing the waveguide for optimum group velocity and third order dispersion, resulting in strong signal generation at $\mathrm{f}(\sim 2.3 \mu \mathrm{m}), 2 \mathrm{f}(\sim 1.15 \mu \mathrm{m})$, and in the C-band ( 1530-1565nm), as shown in Fig.2. See [3] for detail.

\section{Photonics arm}

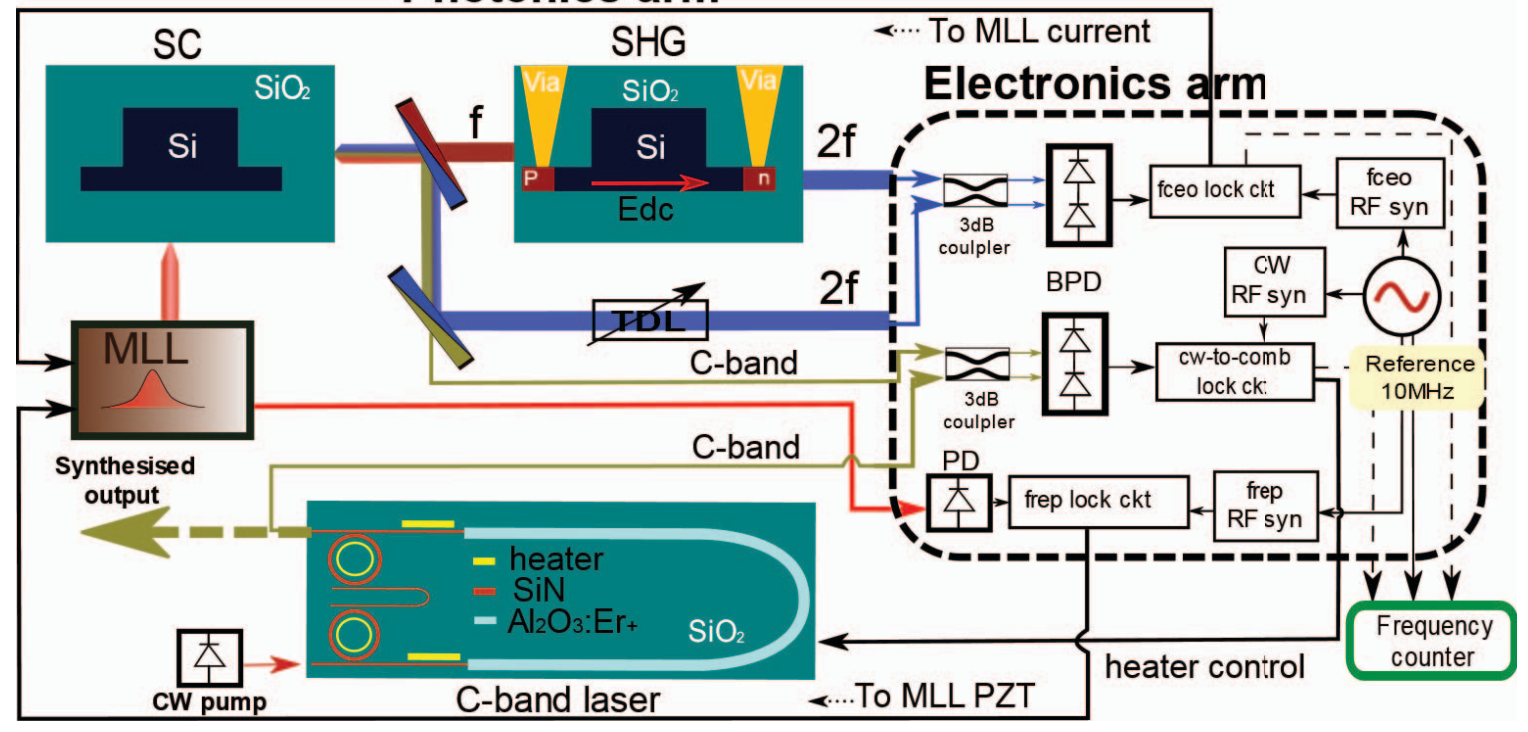

Fig. 1 The silicon photonic synthesizer circuit with its photonics and electronics arms. The MLL is being stabilized with the help of SC generation and SHG in waveguides (see text for detail). The color and black arrows represent optical and electronic signals respectively. The p- and n-type contacts are used to reverse bias the junction across the SHG waveguide using the metal vias. The CW laser is pumped with a $980 \mathrm{~nm}$ diode laser. The solid line from the $10 \mathrm{MHz}$ reference is to synchronize the respective circuit. The dashed line from $\mathrm{f}_{\text {ceo }}$ lock circuit (ckt) and $\mathrm{CW}$-to-comb lock circuit to the frequency counter is indicating measurement. The synthesized optical signal is indicated as dashed arrow from the CW laser. 
The optical synthesizer contains three locking loops, $f_{\text {rep }}, f_{\text {ceo }}$ and CW-to-comb locking. For $f_{\text {rep }}$ locking, the $5^{\text {th }}$ harmonic of the MLL is directly detected by a photodetector (PD) and then compared with a RF synthesizer $\left(f_{\text {rep }} \mathrm{RF}\right.$ syn) to generate the phase error signal, which is fed back to MLL PZT. To detect $f_{\text {ceo, }}$ f-to- $2 \mathrm{f}$ interferometry is required. The f part of the SC signal is sent through a dichroic mirror into the SHG waveguide where it is quasi-phase matched to the $2 \mathrm{f}$ signal being produced by periodically applying DC field orthogonal to the optical field propagation direction in the waveguide. The SHG conversion efficiency is $13 \% / \mathrm{W}$ under $-21 \mathrm{~V}$ DC application [4]. The upconverted $2 \mathrm{f}$ signal and the $2 \mathrm{f}$ part of the SC signal are combined in a 50:50 coupler and mixed in a balanced photodetector (BPD), which can maximize $f_{\text {ceo }}$ and suppress $f_{\text {rep }}$ (by $>20 \mathrm{~dB}$ ) simultaneously. A tunable delay line (TDL) is used in one of the $2 \mathrm{f}$ arms to ensure maximum temporal overlap of the two $2 \mathrm{f}$ pulses so as to maximize the SNR of the $f_{\text {ceo. }}$ The measured $f_{\text {ceo }}$ signal shown in the inset of Fig. 2 (b) is stabilized by feedback to the MLL pump current using a similar

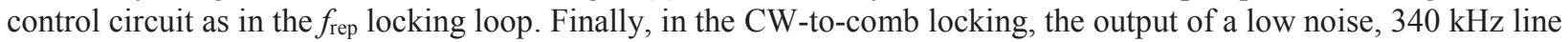
width, C-band laser, which was fabricated by depositing erbium-doped aluminum oxide $\left(\mathrm{Al}_{2} \mathrm{O}_{3}: \mathrm{Er}^{+}\right)$on a silicon nitride on silicon waveguide [5], is beat against the C-band signal (1560nm) of the SC using another 50:50 coupler and BPD. The generated phase error signal from the control electronics is fed to the integrated heater of the $\mathrm{CW}$ laser with $\sim 10 \mu$ s response time. The RF syn's in all three locking loops are locked to the same $10 \mathrm{MHz}$ reference, which guarantee that the final optical synthesizer output is also locked to this RF reference.
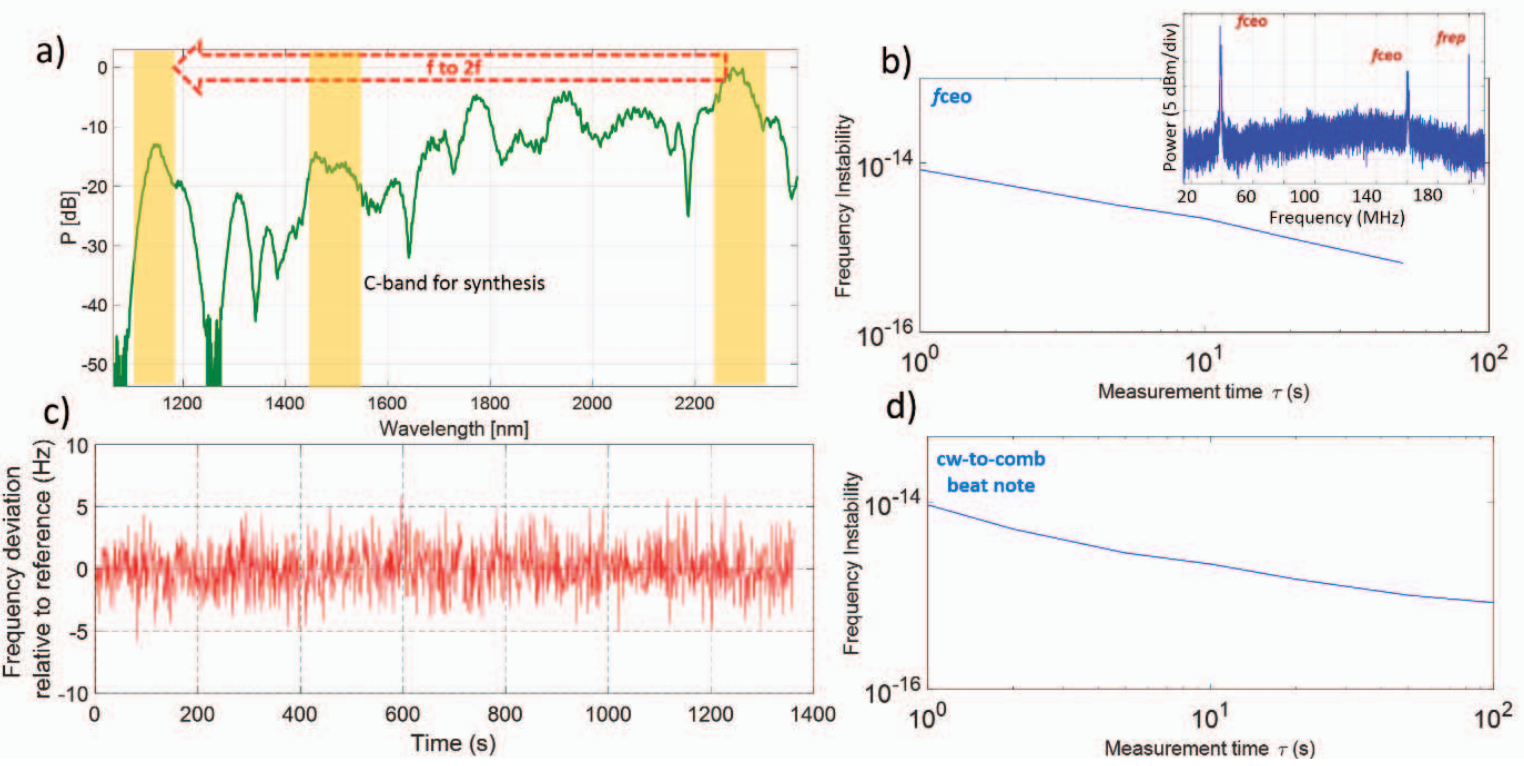

d)

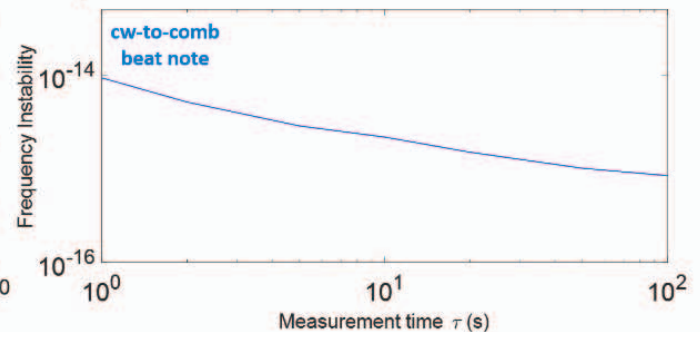

Fig. 2 a) The SC spectrum with the f, $2 \mathrm{f}$ and C-band regions shown in yellow band. b) Measured $f_{\text {ceo }}$ signal (inset) and its frequency instability. c) Frequency deviation of the stabilized $\mathrm{CW}$ laser relative to the optical comb frequency to which it is locked. d) The frequency instability of the CW-to-comb beat note.

The whole synthesizer was successfully operated for over 20 minutes (Fig.2c). The frequency instability (Allan deviation) for the $f_{\text {ceo }}$ and cw-to-comb beat note relative to the $10 \mathrm{MHz}$ reference are shown in Fig. $2 \mathrm{~b}$ and d, both of which are $<1 \times 10^{-14} @ 1$ s and $<1 \times 10^{-15} @ 100$ s. The instability performance of $f_{\text {rep }}$ is much better due to the high SNR in the PD detection. Therefore, a frequency instability of $<1 \times 10^{-15} @ 100$ s can be guaranteed for the optical synthesizer.

In conclusion, we have demonstrated a silicon photonics based frequency synthesizer with a relative frequency instability $<1 \times 10^{-15}$ at $100 \mathrm{~s}$. Future work will include integration of the MLL and exclude coupling loss compensating amplifiers (Tm:ZBLAN for $\mathrm{f}$ amplification and EDFA for the CW laser). Our work leads to various applications including optical communication, spectroscopy, metrology, and integrated atomic clocks. This work was supported under the DARPA DODOS project, contract number HR0011-15-C-0056.

1. D. J. Jones, et. al. "Carrier-Envelope Phase Control of Femtosecond Mode-Locked Lasers and Direct Optical Frequency Synthesis," Science 288, 635-639 (2000).

2. J. D Jost, et. al. "Continuously tunable, precise, single frequency optical signal generator," Opt. Express 20, 515-520 (2002).

3. N. Singh, et. al. "Octave-spanning coherent supercontinuum generation in silicon on insulator from 1.06 $\mu \mathrm{m}$ to beyond $2.4 \mu \mathrm{m}$," Light 7, 1-8 (2018).

4. E. Timurdogan, et. al. "Electric Field-Induced Second Order Nonlinear Optical Effects in Silicon Waveguides," Nat. Photon. 11, 200206 (2017).

5. N. Li, et.al. "CMOS-compatible Tunable Vernier Ring Laser using Erbium Doped Waveguide on a Silicon Photonics Platform" submitted to CLEO (2018). 